\title{
DIVERSIDADE E ÍNDICE SUCESSIONAL DE UMA VEGETAÇÃO DE CERRADO SENSU STRICTO NA UNIVERSIDADE ESTADUAL DE GOIÁS-UEG, CAMPUS DE ANÁPOLIS ${ }^{1}$
}

\author{
Adriana Rosa Carvalho² e Stéphany Marques-Alves ${ }^{3}$
}

\begin{abstract}
RESUMO - A composição vegetal, estrutura, diversidade, estádio sucessional e distribuição de espécies do cerrado do campus da UEG com 6 ha, foram inventariados usando 30 parcelas com $100 \mathrm{~m}^{2}$ cada. A partir de dbs superior ou igual a $5 \mathrm{~cm}$ foram amostrados 515 indivíduos, representados por 20 famílias, 28 gêneros e 46 espécies. As famílias de maior riqueza foram Leguminosae, Vochysiaceae e Malpighiaceae. As espécies Qualea grandiflora, Byrsonima crassa, Erytroxylum tortuosum, Qualea parviflora e Miconia ferruginata apresentaram os maiores VIs. A diversidade da área $\left(\mathrm{H}^{\prime}=1,353\right)$ é menor que valores descritos para Cerrado, o que pode ser conseqüência tanto da abundância de espécies como Q. grandiflora e B. crasssa quanto de interferências antrópicas no local, incluindo queimadas. O valor do índice sucessional $(I S=2,3)$ indica uma comunidade em estágio intermediário de sucessão, já que seu valor oscila de 1 a 3 . A comunidade ajustou-se apenas ao modelo log-normal, o que, de acordo com a literatura, pode ser influência da proporção da abundância de espécies dominantes, intermediárias e raras.
\end{abstract}

Palavras-chave: Diversidade; índice sucessional e cerrado.

\section{DIVERSITY AND SUCCESSIONAL INDEX OF CERRADO SENSU STRICTO AT THE UEG CAMPUS}

\begin{abstract}
This work assessed the composition, diversity, successional stage and distribution of species in 6 ha of cerrado at the UEG campus. Thirty plots $100 \mathrm{~m}^{2}$ each and dbs higher or equal to $5 \mathrm{~cm}$ were used. Of 515 specimens distributed in 20 families, 28 genus and 46 species were sampled. Greater richness was observed in Leguminosae, Vochysiaceae and Malpighiaceae. The species Qualea grandiflora, Byrsonima crassa, Erytroxylum tortuosum, Qualea parviflora and Miconia ferruginata presented higher VIs. Diversity index $(H \phi=1.35)$ was lower than the other values described for the cerrado. This could be a consequence of the abundance of species such as $\mathbf{Q}$. grandiflora and B. crasssa as well as of local anthropogenic interferences, including fire. The value of successional index $(S I=2.3)$ indicates an intermediary stage of succession. The community fitted only to the normal-log model, which, according to the literature, could be a consequence of the abundance proportion of the dominant, intermediary and rare species in the community.
\end{abstract}

Keywords: Diversity; successional index and cerrado.

\footnotetext{
${ }^{1}$ Recebido em 30.05.2006 e aceito para publicação em 18.12.2007.

${ }^{2}$ Laboratório de Biodiversidade do Cerrado da Universidade Estadual deGoiás(UEG), Anapolis-GO. E-mail : <adriana.carvalho@pq.cnpq.br> .

${ }^{3}$ Graduação em Biologia da UEG. E-mail : <stephany.a@bol.com.br>.
} 


\section{INTRODUÇÃO}

Os estudos fitossociológicos surgiram da necessidade de se fornecerem dados a respeito das comunidades vegetais dos diferentes biomas e descrever sua composição, estrutura, distribuição e dinâmica das espécies (GENTRY, 1982; FELFILI e VENTUROLI, 2000).

Ao longo do tempo, inventários fitossociológicos passaram a utilizar os índices de riqueza de espécies, modelos de abundância e índices baseados na abundância proporcional, a fim de auxiliar a compreensão da estrutura da comunidade (FELFILI e VENTUROLI, 2000; CARVALHO, 2003). Particularmente, os modelos de abundância, embora ainda sejam pouco utilizados, permitem descrever matematicamente os dados e obter inferências da estrutura e riqueza da vegetação (MAGURRAN, 1988; FELFILI e VENTUROLI, 2000). Recentemente, a abordagem quantitativa em estudos fitossociológicos tem sido usada na compreensão da composição vegetal e de seu estádio sucessional (PETRERE et al., 2004).

O Cerrado sensu stricto ocupa cerca de $70 \%$ do bioma cerrado (EITEN, 1994), e os estudos fitossociológicos e florísticos já realizados neste bioma indicam grande diversidade fitofisionômica e uma disposição em matriz vegetacional. De acordo com Ratter e Dargie (1992) e Ribeiro e Walter (1998), essa grande heterogeneidade é determinada por aspectos edáficos, latitude, freqüentes queimadas e fatores antrópicos, que são capazes de influenciar a fertilidade do solo e o crescimento da vegetação, bem como a distribuição das espécies vegetais (NASCIMENTO e SADDI, 1992).

Do ponto de vista florístico, essa vegetação é formada por arbustos, arvoretas e um estrato herbáceo dominado por gramíneas. A vegetação lenhosa é caracterizada por possuir troncos e galhos torcidos, às vezes inclinados (RIBEIRO e WALTER, 1998).

A partir de 1960, devido a interiorização da capital do país para Brasília e do processo de transformação do uso do solo no bioma Cerrado, houve intensa alteração nesse bioma. Nesse período, o Brasil teve sua fronteira agrícola rapidamente deslocada em direção ao centronorte, resultando em perda ou modificação de $67 \%$ da área do Cerrado (NÓBREGA e ENCINAS, 2006).

Como resultado, houve descaracterização da composição da vegetação nativa e redução na cobertura vegetal em pequenos fragmentos (ALHO, 1990; AGUIAR et al., 2004; MARRIS, 2005), com a conseqüente introdução de espécies exóticas que causaram o declínio da biodiversidade nesse bioma (MACHADO et al., 1999; DINIZ-FILHO et al., 2004) de forma que restam, hoje, 2,2\% de sua área original protegida, e houve sua quase eliminação na Região Sudeste.

Estudos realizados em Cerrado de Goiás e do Distrito Federal revelam alto índice de diversidade e grande heterogeneidade fitofisionômica nessas formações, com predominância da família Leguminosae (RIBEIRO e WALTER, 1998; SILVA et al., 2002; SAPORETTI JR et al., 2003; ASSUNÇÃO e FELFILI, 2004).

Assim, com o intuito de usar dados fitossociológicos para estudar a estrutura de uma vegetação de cerrado no campus da UEG foram utilizados modelos de distribuição de espécies e índices que estimam a diversidade e o estádio sucessional desta comunidade.

\section{MATERIAL E MÉTODOS}

\section{1. Área de estudo}

O estudo foi realizado no Campus da Universidade Estadual de Goiás, entre as coordenadas $16^{\circ} 20^{\prime} 34^{\prime \prime} \mathrm{S}$ e $48^{\circ} 52^{\prime} 51^{\prime \prime} \mathrm{W}$ e $997 \mathrm{~m}$ de altitude, situada no Município de Anápolis, no Estado de Goiás. Por ser uma área de Cerrado, seu clima predominante é o tropical sazonal, de inverno seco e verão chuvoso. Sua média de precipitação anual fica entre 1.200 e 1.800 mm e temperatura média anual em torno de $22-23^{\circ} \mathrm{C}$ (COUTINHO, 2000).

A vegetação do local é composta basicamente por cerrado sensu stricto, Mata de Transição e Mata Ciliar. A análise fitossociológica compreendeu a área coberta por Cerrado, que se estendia originalmente por 10 ha e hoje ocupa cerca de 7 ha.

\subsection{Análise fitossociológica e de distribuição de espécies}

Para testar a hipótese nula principal de que a estrutura fitossociológica é constituída de espécies igualmente distribuídas, foi utilizado o método de parcelas com área de $100 \mathrm{~m}^{2}$ cada uma $(10 \times 10 \mathrm{~m})$, distribuídas em transecções na área cortada pela trilha de Educação Ambiental, ao longo da vegetação de Cerrado sensu stricto. Ao todo foram delimitadas 30 parcelas. Cada extremidade foi marcada com estacas de madeira, e uma delas foi enumerada para permitir a identificação. O tamanho adotado nas parcelas, embora não haja um padrão definido, seguiu o mesmo utilizado em outros estudos sobre Cerrado (FERREIRA et al., 1993; TEIXEIRA et al., 2004). 
Para avaliar a suficiência do número de parcelas, foi utilizada a curva do coletor, ou seja, o $\mathrm{N}$ amostral (número de parcelas) foi definido quando as últimas parcelas não mais acrescentaram novas espécies à amostragem.

Foram amostrados e numerados com plaquetas de alumínio todos os indivíduos vivos ou mortos, ainda em pé, com diâmetro à base do solo (dbs) superior ou igual a $5 \mathrm{~cm}$ (van den BERG e OLIVEIRA-FILHO, 2000). Os indivíduos mortos ainda em pé, embora não identificados, foram classificados estatisticamente como uma só família, seguindo-se a recomendação de Teixeira et al. (2004).

Nos casos em que houve indivíduos de espécies iguais com distância entre caules inferior ou igual a 30 cm, os dbs's (diâmetro à base do solo) dos troncos/ caules foram somados e, quando atingisse $5 \mathrm{~cm}$, o exemplar era incluído na amostragem como um único indivíduo, tal qual o sugerido por Pagano et al. (1995).

As amostragens em campo foram realizadas de janeiro/05 a agosto/05 para registro de altura, dbs e o nome científico ou popular das espécies, se identificadas. As espécies não reconhecidas ou não presentes no herbário da instituição foram coletadas para montagem de exsicata e posterior identificação. O sistema de classificação adotado foi o de Cronquist (1981), com exceção da família Leguminosae, em que se usou o sistema de classificação de Engler (1964), segundo o sugerido por Rocha e Muniz (1998).

Para a análise fitossociológica foram determinados os seguintes parâmetros: número de indivíduos por espécie, os valores absolutos e relativos de densidade, freqüência e dominância, o índice de importância e o índice de cobertura (FELFILI e VENTUROLI, 2000). No cálculo da diversidade da comunidade vegetal utilizouse o Índice de Shannon- Wiener $\left(\mathrm{H}^{\prime}\right)$

$$
H^{\prime}=\sum\left(\frac{n_{i}}{N}\right) \times \log \frac{n_{i}}{n}
$$

em que: $\mathrm{H}^{\prime}=$ índice de diversidade de Shannon-Wiener; ni= número de indivíduos da espécie $i$; e $\mathrm{N}=$ número total de indivíduos.

A eqüitabilidade $(J)$ foi calculada pela seguinte fórmula:

$$
\mathrm{J}=\mathrm{H}^{\prime} / \log \mathrm{S}
$$

em que: $\mathrm{H}^{\prime}$ = índice de diversidade de Shannon-Wiener e $\mathrm{S}=$ número total da amostra.
Na estimativa da diversidade levando em consideração o estádio sucessional em que a comunidade vegetal se encontra, foi utilizado o Índice Sucessional - SI, descrito por Petrere et al. (2004).

$$
S I=\sum w_{i} \times\left(\frac{n_{i}}{N}\right)
$$

em que: $\mathrm{Wi}$ = categoria sucessional de cada espécie; ni= número de indivíduos da espécie $i$; e $\mathrm{N}=$ número total de indivíduos.

A distribuição das espécies foi avaliada através do ajuste dos dados aos modelos de espécie-abundância (broken-stick, log-normal, log-série e série geométrica), com comparação das curvas teóricas com as observadas através do teste do qui-quadrado $\left(\chi^{2}\right)$.

\section{RESULTADOS}

No total foram amostrados 515 indivíduos, dos quais nove estavam mortos ainda em pé. Os demais foram representados por 20 famílias, 28 gêneros e 46 espécies; dentre estas últimas, nove não foram identificadas. Esses indivíduos e a classe sucessional indicada na literatura são apresentados na Tabela 1.

Do total de indivíduos amostrados, 79\% apresentaram dbs menor que $10 \mathrm{~cm}$ e altura menor que 1,9 m. A altura média dos indivíduos amostrados foi de 1,49 m (máxima $=4$ m; mínima $=0,17$ m; e d.p. $=61,48$ m). O maior diâmetro obtido foi de um espécime de Qualea grandiflora com 32,80 cm e o diâmetro médio de 8,09 cm (d.p. = 4,25 cm). A distribuição de altura e dbs apresentou a forma de $\mathrm{J}$ invertido (Figura 1).

A família Leguminosae teve o maior número de espécies (n=7) e representa 20,5\% do total. Vockysiaceae e Malpighiaceae, com cinco e três espécies, respectivamente, resultando em $23,5 \%$ do total de espécies amostradas (Tabela 2). As famílias Compositae, Erytroxylaceae, Guttiferae e Nyctaginaceae contribuíram com duas espécies cada uma. As 14 famílias restantes foram representadas por apenas uma espécie cada uma (Tabela 1). Vochysiaceae ( $n=101)$, Malpighiaceae $(n=85)$ e Erytroxylaceae $(n=74)$ foram as famílias mais abundantes na área (Tabela 2).

A espécie de maior abundância (Figura 2) foi Byrsonima crassa com 76 indivíduos, compreendendo $14 \%$ do total de indivíduos $(\mathrm{n}=515)$. Q. grandiflora é a segunda espécie em abundância na área, com 55 indivíduos, representando 10,5\% do total. As três

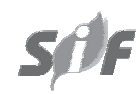

R. Árvore, Viçosa-MG, v.32, n.1, p.81-90, 2008 
seguintes foram Erytroxylum tortuosum (n=50; 9,6\%), Qualea parviflora ( $\mathrm{n}=40 ; 7,7 \%)$ e Miconia ferruginata $(\mathrm{n}=37 ; 7,1 \%)$.

Em relação à densidade, $B$. crassa representou $14 \%$ do total, seguida de Q. grandiflora com $10 \%$. Mimosa claussenii, Aspidosperma macrocarpon, Kielmeyera rubriflora, Vochysia thyrsoidea e outras seis espécies não identificadas contribuíram com apenas um indivíduo e tiveram densidade relativa de 0,19 .

As cinco espécies com maiores freqüências relativas foram B. crassa, Q. grandiflora, Erytroxylum suberosum, Q. parviflora e M. ferruginata, que juntas representaram $36,7 \%$ do total.

Tabela 1 - Famílias, espécies arbustivo-arbóreas e estádio sucessional utilizado no estudo fitossociológico em área de cerrado situada no campus da UEG em Anápolis, GO ( $2^{\mathrm{a}}$ tardia = secundária tardia; c.a.d .= colonizadora de áreas degradadas, e c.c. = característica de cerrado)

Table 1 - Families, species and successional stage used in the phyto-sociological study in area of cerrado sensu stricto at the UEG campus in Anápolis-GO (later secondary; c.a.d.=colonizer of impacted areas; c.c. = typical of cerrado)

\begin{tabular}{|c|c|c|c|}
\hline Família & Espécie & Nome popular & Estádio sucessional \\
\hline Apocynaceae & Aspidosperma macrocarpon Mart. & Peroba-do-cerrado & $2^{a}$ tardia/climax \\
\hline Araliaceae & $\begin{array}{l}\text { Scheflera macrocarpa } \\
\text { (Cham. e Schltdl) Frodim }\end{array}$ & Mandiocão-do-cerrado & pioneira (c.a.d.) \\
\hline Bombacaceae & $\begin{array}{l}\text { Eriotheca pubescens (Mart. e Zucc.) } \\
\text { Schott e Endl. }\end{array}$ & Paineira-do-campo & $2^{\mathrm{a}}$ tardia/climax \\
\hline Celastraceae & Plenkia populnea Reissek & Marmelo-do-cerrado & não encontrado \\
\hline Compositae & $\begin{array}{l}\text { Piptocarpha rotundifolia (Less.) Baker } \\
\text { Eremanthus glomerulatus Less. }\end{array}$ & $\begin{array}{l}\text { Coração-de-negro } \\
\text { Coração-de-negro } \\
\end{array}$ & $\begin{array}{ll}\text { pioneira } & \text { (c.a.d.) } \\
\text { pioneira } & \text { (c.a.d.) } \\
\end{array}$ \\
\hline Dilleniaceae & Davilla elliptica A. St.- Hil. & Lixeirinha & clímax (c.c.) \\
\hline Erytroxylaceae & $\begin{array}{l}\text { Erytroxylum suberosum A. St.- Hil. } \\
\text { Erytroxylum tortuosum Mart. }\end{array}$ & $\begin{array}{l}\text { Cabelo-de-negro } \\
\text { Muxiba-comprida }\end{array}$ & $\begin{array}{l}\text { clímax (c.c.) } \\
2^{\mathrm{a}} \text { tardia/climax }\end{array}$ \\
\hline$\overline{\text { Guttiferae(Clusiaceae) }}$ & $\begin{array}{l}\text { Kielmeyera coriacea (Spreng.) Mart. } \\
\text { Kielmeyera rubriflora }\end{array}$ & $\begin{array}{l}\text { Pau-santo } \\
\text { Pau-santo } \\
\end{array}$ & $\begin{array}{l}\text { clímax (c.c.) } \\
\text { clímax (c.c.) }\end{array}$ \\
\hline Leguminosae & $\begin{array}{l}\text { Dalbergia miscolobium Benth. } \\
\text { Dimorphandra mollis Benth. } \\
\text { Hymenaea stigonocarpa Mart. ex Hayne } \\
\text { Machaerium opacum Vogel } \\
\text { Mimosa claussenii Benth. } \\
\text { Stryphnodendron adstringens (Mart.) Coville } \\
\text { Stryphnodendron polyphilum }\end{array}$ & $\begin{array}{l}\text { Jacarandá-do-cerrado } \\
\text { Faveira-do-campo } \\
\text { Jatobá-do-cerrado } \\
\text { Jacarandá-cascudo } \\
\text { Mimosa } \\
\text { Barbatimão } \\
\text { Barbatimão } \\
\end{array}$ & $\begin{array}{l}\text { pioneira } \\
\text { pioneira } \\
2^{\text {a }} \text { tardia/climax } \\
\text { pioneira } \\
\text { pioneira (c.a.d.) } \\
\text { pioneira } \\
\text { formações } 2^{\text {as }} \\
\end{array}$ \\
\hline Lythraceae & Lafoensia pacari A. St.- Hil. & Pacari & pioneira \\
\hline Malpighiaceae & $\begin{array}{l}\text { Byrsonima crassa Nied } \\
\text { Byrsonima coccolobifolia H. B. e K. } \\
\text { Byrsonima verbacifolia (L.) DC. }\end{array}$ & $\begin{array}{l}\text { Murici } \\
\text { Murici-rosa } \\
\text { Muricizão } \\
\end{array}$ & $\begin{array}{l}\text { clímax (c.c.) } \\
\text { clímax (c.c.) } \\
\text { pioneira } / 2^{\mathrm{a}} \text { inicial } \\
\end{array}$ \\
\hline Melastomataceae & Miconia ferruginata DC. & Pixirica & clímax (c.c.) \\
\hline Myrsinaceae & Rapanea guianensis Aubl. & Cafezinho & formaçoes $2^{\mathrm{a}}$ \\
\hline Nyctaginaceae & $\begin{array}{l}\text { Guapira noxia (Netto) Lundell } \\
\text { Neea theifera Oerst. }\end{array}$ & $\begin{array}{l}\text { Caparrosa } \\
\text { Caparrosa-branca }\end{array}$ & $\begin{array}{l}2^{\mathrm{a}} \text { tardia/climax } \\
\text { clímax (c.c.) }\end{array}$ \\
\hline Ochanaceae & Ouratea hexasperma (A. St.- Hil.) Baill. & Vassoura-de-bruxa & não encontrada \\
\hline $\begin{array}{l}\text { Proteaceae } \\
\end{array}$ & Roupala montana Aubl. & Carne-de-vaca & clímax (c.c.) \\
\hline Rubiaceae & Palicourea rígida Kunth. & Bate-caixa & clímax (c.c.) \\
\hline Styracaceae & Styrax ferrugineus Nees e Mart. & Laranjinha-do-cerrado & clímax (c.c.) \\
\hline Velloziaceae & Vellozia sp & Canela-de-ema & clímax (c.c.) \\
\hline Vochysiaceae & $\begin{array}{l}\text { Qualea grandiflora Mart. } \\
\text { Qualea parviflora Mart. } \\
\text { Qualea multiflora Mart. } \\
\text { Vochysia cinamomea } \\
\text { Vochysia thyrsoidea Pohl }\end{array}$ & $\begin{array}{l}\text { Pau-terra-grande } \\
\text { Pau-terra-roxo } \\
\text { Pau-terra-liso } \\
\text { Pau-doce } \\
\text { Gomeira }\end{array}$ & $\begin{array}{l}\text { formações } 1^{\text {as }} \text { e } 2^{\text {as }} \\
\text { clímax (c.c.) } \\
\text { pioneira } \\
\text { pioneira } \\
\text { pioneira }\end{array}$ \\
\hline
\end{tabular}



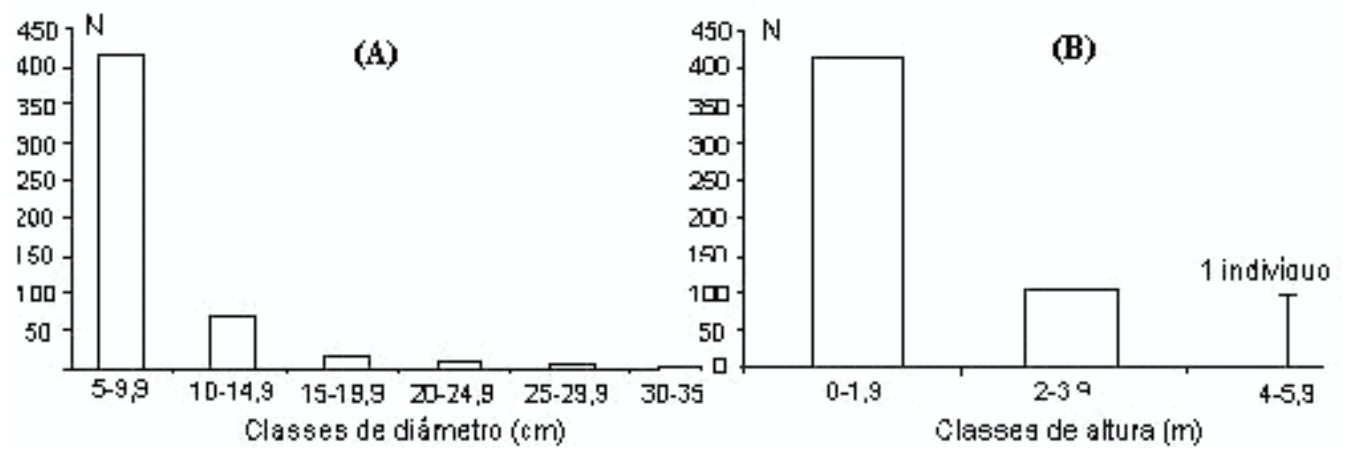

Figura 1 - Distribuição dos indivíduos por classes de diâmetros (A) e classes de altura (B) no cerrado sensu stricto, no campus da UEG, em Anápolis, GO.

Figure 1 - Distribution of the individuals per diameter classes (A) and height classes (B) in the cerrado sensu stricto at the UEG campus in Anápolis, GO.

Q. grandiflora e B. crassa representaram $41 \%$ do total de dominância. Ambas as espécies tiveram valores discrepantes em relação às demais abundâncias encontradas nas outras espécies. Essa discrepância também foi notada quanto ao VC (valor de cobertura), que representou 34,9 para Q. grandiflora e 31,6 para B. crassa. E. suberosum, apesar de ser a terceira espécie em VC, tem a metade do valor das duas primeiras ( $\mathrm{VC}=16,3$ ).

Seguindo o padrão de abundâncias, densidades e freqüências, as espécies Q. grandiflora, B. crassa,E. tortuosum, Q. parviflora e M. ferruginata apresentaram os maiores VI, contribuindo com 49,8\% do total (Tabela 2 e Figura 2).

O índice de diversidade de Shannon-Wiener $\left(\mathrm{H}^{\prime}\right)$ estimado para a área estudada foi de 1,353, considerado baixo em relação aos valores descritos sobre o Cerrado. O valor encontrado para o índice sucessional foi de 2,3 .

A comunidade ajustou-se apenas ao modelo de log-normal (Figura 3 ), uma vez que o valor do quiquadrado $\left(\chi^{2}=3,76, \mathrm{P}=0,000\right)$ foi menor que o tabelado $\left(\chi^{2}=16,9\right)$. Nenhum dos demais modelos se adaptou aos dados.

\section{DISCUSSÃO}

Franco (2004), utilizando o método de caminhamento para levantamento florístico (de herbáceas a arbustivas) na mesma área aqui estudada, registrou a ocorrência de 64 espécies. No levantamento fitossociológico aqui descrito, houve a ocorrência de um número menor de espécies ( $S=46$ ), possivelmente em conseqüência da metodologia utilizada e da estrutura avaliada, visto que só espécies arbóreas foram estudadas.

Ratter et al. (1992) e Ribeiro e Walter (1998), ao compararem 98 áreas de Cerrado sensu stricto, relacionaram 26 espécies arbóreas comuns em pelo menos 50\% das áreas. Dessa lista de espécies, 12 ocorreram também neste estudo (Byrsonima coccolobifolia, Byrsonima verbascifolia, Dimorphandra mollis, E. suberosum, Hymenea stigonocarpa, Kielmeyera coriacea, Lafoensia pacari, Q. grandiflora, Qualea multiflora, Q.parviflora, Roupala Montana e Tabebuia ochracea), com destaque para Q. grandiflora e para o gênero Byrsonima, que foram muito abundantes na área de estudo deste trabalho.

As espécies $M$. ferruginata, Ouratea hexasperma, Piptocarpha rotundifolia e Scheflera macrocarpa que de acordo com Ribeiro e Walter (1998) são características do cerrado sensu stricto também ocorreram na área do cerrado estudado na UEG.

A média das alturas estimadas encontradas nesse estudo $(1,5 \mathrm{~m})$ difere de outros trabalhos realizados em áreas de cerrado sensu stricto que varia entre 2 e 3 m (RIBEIRO et al., 1985; NASCIMENTO e SADDI, 1992; SAPORETTIJR etal., 2003; ASSUNÇÃO e FELFILI, 2004). A existência desse baixo estrato arbóreo indica que a vegetação dessa área é composta basicamente por indivíduos jovens. Isso é confirmado pela distribuição das alturas e diâmetros em forma de J invertido, que resulta na presença da maioria dos indivíduos amostrados (cerca de 80\%) na primeira classe de distribuição (Figura 2).

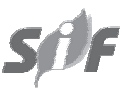

R. Árvore, Viçosa-MG, v.32, n.1, p.81-90, 2008 
Tabela 2 - Parâmetros fitossociológicos (ordem crescente de VI) das espécies amostradas no cerrado do campus da UEG, em Anápolis, GO

Table 2 - Species and phytosociological parameters of cerrado sensu stricto at UEG campus in Anápolis

\begin{tabular}{|c|c|c|c|c|c|c|}
\hline Espécie & $\mathrm{n}$ & DR & FR & DoR & VI & VC \\
\hline Qualea grandiflora & 55 & 10,597 & 7,666 & 24,383 & 42,620 & 34,981 \\
\hline Byrsonima crassa & 76 & 14,644 & 8,711 & 17,046 & 40,370 & 31,690 \\
\hline Erytroxylum tortuosum & 50 & 9,634 & 6,969 & 6,755 & 23,334 & 16,389 \\
\hline Qualea parviflora & 40 & 7,707 & 6,969 & 6,562 & 21,214 & 14,269 \\
\hline Miconia ferruginata & 37 & 7,129 & 6,620 & 7,216 & 20,943 & 14,346 \\
\hline Erytroxylum suberosum & 34 & 6,551 & 6,272 & 3,552 & 16,353 & 10,103 \\
\hline Eremanthus glomerulatus & 24 & 4,624 & 5,923 & 2,654 & 13,181 & 7,278 \\
\hline Palicourea rigida & 20 & 3,854 & 5,575 & 2,670 & 12,080 & 6,524 \\
\hline Stryphnodendron adstringens & 16 & 3,083 & 3,833 & 3,007 & 9,909 & 6,090 \\
\hline Vellozia sp, & 13 & 2,505 & 3,136 & 2,474 & 8,103 & 4,978 \\
\hline Kielmeyera coriacea & 11 & 2,119 & 2,787 & 2,366 & 7,263 & 4,485 \\
\hline Davilla elliptica & 12 & 2,312 & 2,091 & 1,386 & 5,781 & 3,698 \\
\hline Piptocarpha rotundifolia & 9 & 1,734 & 1,394 & 2,593 & 5,716 & 4,328 \\
\hline Roupala montana & 10 & 1,927 & 2,091 & 1,197 & 5,207 & 3,123 \\
\hline ni 1 & 9 & 1,734 & 1,742 & 1,711 & 5,182 & 3,445 \\
\hline Lafoensia pacari & 8 & 1,541 & 2,439 & 0,833 & 4,805 & 2,374 \\
\hline Nea theyfereae & 10 & 1,927 & 1,742 & 1,062 & 4,725 & 2,989 \\
\hline Mortas ainda em pé & 9 & 1,734 & 1,742 & 1,151 & 4,622 & 2,886 \\
\hline Styrax ferrugineus & 5 & 0,963 & 1,742 & 1,317 & 4,017 & 2,281 \\
\hline Ouratea hexasperma & 7 & 1,349 & 2,091 & 0,569 & 4,001 & 1,918 \\
\hline Rapanea guianensis & 6 & 1,156 & 1,394 & 1,213 & 3,758 & 2,369 \\
\hline Guapira noxia & 6 & 1,156 & 1,742 & 0,607 & 3,499 & 1,763 \\
\hline Dalbergia miscolobium & 3 & 0,578 & 1,045 & 1,818 & 3,438 & 2,396 \\
\hline Byrsonima verbacifolia & 5 & 0,963 & 1,394 & 0,541 & 2,894 & 1,505 \\
\hline Byrsonima coccolobifolia & 4 & 0,771 & 1,394 & 0,432 & 2,591 & 1,202 \\
\hline Machaerium opacum & 4 & 0,771 & 1,045 & 0,464 & 2,276 & 1,235 \\
\hline Scheflera macrocarpa & 4 & 0,771 & 1,045 & 0,359 & 2,171 & 1,130 \\
\hline Qualea multiflora & 3 & 0,578 & 1,045 & 0,312 & 1,932 & 0,890 \\
\hline Stryphnodendron polyphilum & 4 & 0,771 & 0,697 & 0,314 & 1,779 & 1,085 \\
\hline Vochysia cinamomea & 2 & 0,385 & 0,697 & 0,344 & 1,424 & 0,730 \\
\hline ni2 & 2 & 0,385 & 0,697 & 0,244 & 1,324 & 0,630 \\
\hline Dimorphandra mollis & 2 & 0,385 & 0,697 & 0,222 & 1,302 & 0,608 \\
\hline Tabebuia Ochracea & 2 & 0,385 & 0,697 & 0,201 & 1,280 & 0,586 \\
\hline $\mathrm{Ni3}$ & 2 & 0,385 & 0,697 & 0,189 & 1,269 & 0,575 \\
\hline ni 4 & 2 & 0,385 & 0,348 & 0,288 & 1,020 & 0,673 \\
\hline Hymenaea stigonocarpa & 2 & 0,385 & 0,348 & 0,178 & 0,911 & 0,564 \\
\hline Eriotheca pubescens & 1 & 0,193 & 0,348 & 0,696 & 1,236 & 0,889 \\
\hline Mimosa claussenii & 1 & 0,193 & 0,348 & 0,244 & 0,784 & 0,437 \\
\hline ni5 & 1 & 0,193 & 0,348 & 0,152 & 0,692 & 0,345 \\
\hline Aspidosperma macrocarpon & 1 & 0,193 & 0,348 & 0,141 & 0,681 & 0,333 \\
\hline $\mathrm{Ni6}$ & 1 & 0,193 & 0,348 & 0,094 & 0,634 & 0,286 \\
\hline Kielmeyera rubriflora & 1 & 0,193 & 0,348 & 0,085 & 0,625 & 0,277 \\
\hline Vochysia thyrsoidea & 1 & 0,193 & 0,348 & 0,085 & 0,625 & 0,277 \\
\hline ni7 & 1 & 0,193 & 0,348 & 0,085 & 0,625 & 0,277 \\
\hline ni8 & 1 & 0,193 & 0,348 & 0,076 & 0,616 & 0,269 \\
\hline Plenckia populnea & 1 & 0,193 & 0,348 & 0,056 & 0,596 & 0,249 \\
\hline ni 9 & 1 & 0,193 & 0,348 & 0,056 & 0,596 & 0,249 \\
\hline
\end{tabular}

A ocorrência de espécies comuns no estudo de Ratter (1992) e Ribeiro e Walter (1998) e a freqüência de alturas indicam que a vegetação estudada tem características de Cerrado sensu stricto, subtipo Cerrado ralo, segundo a definição de Ribeiro e Walter (1998).

A maior riqueza de espécies da família Leguminosae é confirmada por Mendonça et al. (1998), que a consideram a família mais rica na maioria dos levantamentos fitossociológicos realizados em áreas de Cerrado. Da mesma forma, Heringer e Ferreira (1974) apontaram as Leguminosae como a família de maior riqueza de espécies em ambiente savanícola, afirmação corroborada pelos levantamentos dos próprios autores. 


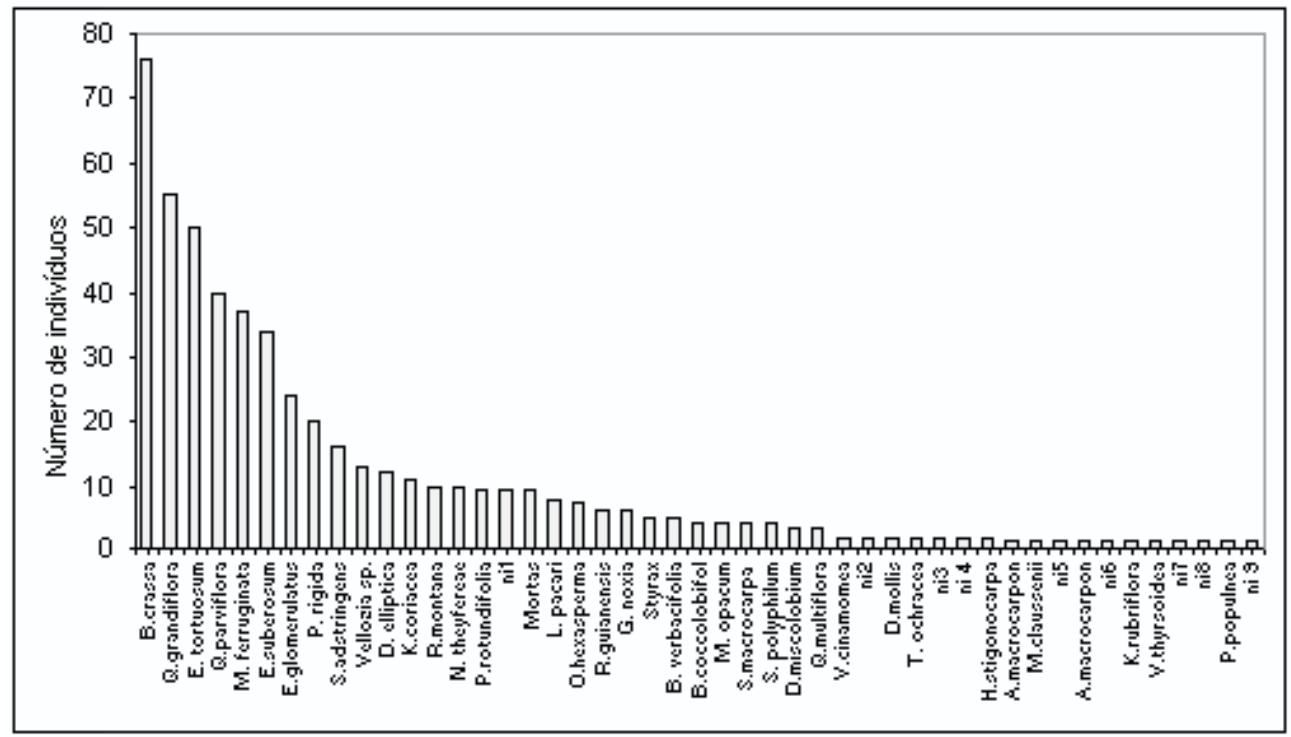

Figura 2 - Distribuição de abundância das espécies amostradas no cerrado do campus da UEG em Anápolis, GO.

Figure 2 - Distribution of abundance of the species sampled in the cerrado at the UEG campus in Anápolis, GO - Brazil.

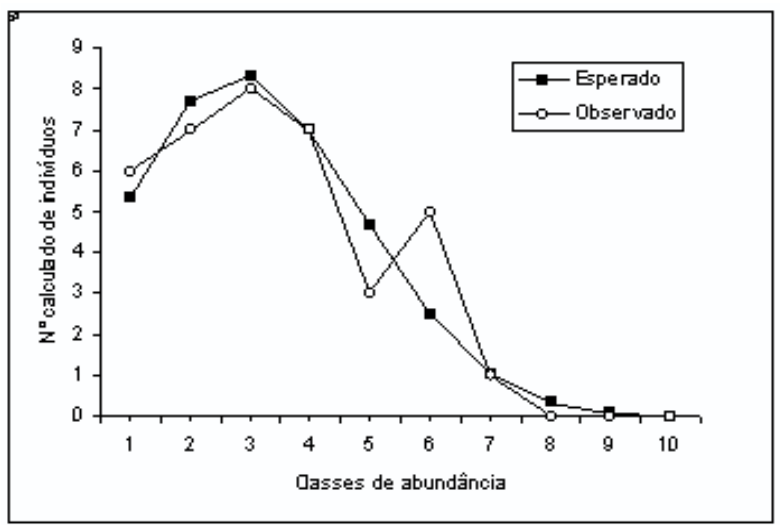

Figura 3 - Comparação entre curva teórica de distribuição log-normal (calculado) com a curva obtida (observado) no estudo feito na área de cerrado do campus da UEG, em Anápolis, GO.

Figure 3 - Comparison between theoretical curve of normallog distribution (calculated) and the curve obtained (observed) in the cerrado surveyed at the UEG campus in Anápolis, GO.

Além de Leguminosae, as famílias Vochysiaceae e Malpighiaceae apresentaram valores altos de riqueza, tal qual o verificado também por Weiser e Godoy (2001), Assunção e Felfili (2004) e Felfili et al. (2002).

A família Vochysiaceae geral é considerada representativa de vegetação de cerrado. Neste estudo, embora Vochysiaceae seja a família mais abundante na área, essa abundância foi representada, principalmente, pelas espécies Q. grandiflora e Q. parviflora, já que Q.multiflora contribuiu apenas com três indivíduos dentro dessa família. Assim, a predominância de $Q$. grandiflora resultou em maior VI, condizente com os resultados encontrados também por Goodland e Ferri (1979) e Teixeira et al. (2004). Essa espécie também está entre as primeiras com maior VI em trabalhos realizados por Silva et al. (2002), Ribeiro et al. (1985) e Ribeiro (1983). A espécie B. crassa, que tem o segundo maior VI neste trabalho, também foi representativa no levantamento realizado por Felfili et al. (2002).

O valor do índice de diversidade de Shannon-Wiener $\left(\mathrm{H}^{\prime}\right)$ foi baixo quando comparado com outros levantamentos em áreas de Cerrado sensu stricto (RIBEIRO et al., 1985; NASCIMENTO e SADDI, 1992; SAPORETTI Jr. et al., 2003; FIDELIS e GODOY, 2003; ASSUNÇÃO e FELFILI, 2004), que em geral apresentam o H' em torno de 3,00. Isso, provavelmente, resulta da distribuição não homogênea de espécies na área, devido à abundância de algumas poucas espécies, como a Q. grandiflora e B. crassa.

Isso refuta a hipótese inicial de distribuição eqüitativa de espécies e determina o valor mais baixo do índice. Outro fator importante é que a área em questão sofre 
fortes influências antrópicas, já que se encontra rodeada pelo campus da universidade, por uma construção civil e por fazendas destinadas a pastagens. Além disso, essa área esteve sujeita a queimadas acidentais três vezes nos últimos seis anos (2000, 2005 e 2006), e isso certamente afetou a abundância de algumas espécies, determinando o baixo valor de H' e a alta dominância.

A comunidade se ajustou ao modelo log-normal, o que, de acordo com a literatura, pode ser evidência de uma comunidade madura e que não sofre efeito de perturbações e distúrbios antrópicos. Porém, dado o nosso conhecimento da área e sabendo que ela sofre distúrbios variados, é mais provável que o ajuste ao modelo log-normal seja resultado de uma amostragem representativa, pois ele é conseqüência do teorema do limite central que prevê que quando um grande número de variáveis atuando sobre determinada medida, no caso a distribuição de espécies; essa medida apresentará distribuição normal ou log-normal.

Além disso, Ugland e Gray (1982) demonstraram que a log-normal é um modelo comum em dados ecológicos, cuja proporção seja em torno de $65 \%$ de espécies raras, 25\% de espécies intermediárias e somente $10 \%$ de espécies abundantes. No Cerrado estudado na UEG, a proporção encontrada (Figura 2) foi de 12,7\% de espécies dominantes, 25,5\% de intermediárias e 61,7\% de espécies raras, o que reforça o ajuste ao modelo citado.

Gray (1987) e Watt (1998) consideraram, no entanto que o padrão de espécies-abundância se ajusta à série geométrica em estágios sucessionais iniciais e, à medida que mais espécies aparecem ao longo da sucessão, a comunidade desenvolve uma distribuição log-normal. O valor do índice sucessional neste estudo $(\mathrm{SI}=2,3)$ indica uma comunidade em estágio intermediário de sucessão, já que seu valor pode variar entre 1 e 3 , ou seja, o índice sucessional atingiria seu valor máximo ( $\mathrm{SI}=3$ ) em áreas que apresentassem apenas espécies tardias, indicando um estágio mais avançado de sucessão; e seu valor mínimo ( $\mathrm{SI}=1$ ) em formações vegetais em início de sucessão, evidencia apenas espécies pioneiras (PETRERE et al., 2004).

De qualquer forma, distúrbios freqüentes (como o desmatamento recente devido à passagem de um trator sobre a vegetação em junho de 2005 e o fogo ocasional a que a área está sujeita) são representativos e alteram a vegetação, sua distribuição de espécies - abundância e estádio sucessional. Além disso, as características estruturais do Cerrado, uma vegetação esparsa, mais aberta e com maior incidência de luz, dificultam o estabelecimento de classes e estádios sucessionais com a mesma precisão que em outras formações (como mata atlântica ou amazônica).

\section{CONCLUSÃO}

A área estudada é um Cerrado sensu stricto cuja fitofisionomia é de Cerrado ralo. Do total de espécies amostradas $(\mathrm{S}=46)$.

Não há distribuição homogênea das espécies na área, já que 11 espécies são de ocorrência rara (apenas um indivíduo), e apenas 14 têm mais de 10 indivíduos. Assim, a maior abundância registrada foi da família Vochysiaceae, de forma que Qualea grandiflora apresentou os maiores VI e VC. Também, a riqueza não é homogênea (maior na família Leguminosae), de forma que o índice de diversidade estimado é baixo. Isso, provavelemente, resulta das interferências antrópicas no local, que incluem a passagem do fogo e podem influenciar o desenvolvimento da comunidade, em especial a dominância de algumas espécies.

Apesar dessas interferências, o índice de estágio sucessional indica que essa formação se encontra numa fase intermediária de sucessão, apresentando espécies de colonizadoras a emergentes. Isso parece ser respaldado pelo ajuste ao modelo log-normal, embora estimativas semelhantes em outras formações de cerrado sejam necessárias para verificar este possível padrão. Estas características podem oferecer um importante arcabouço para estudos futuros que permitam monitorar o estágio sucessional e o índice de diversidade, em especial na área estudada caso seja mantida a atual vigilância para evitar queimadas e outras atividades que possam suprimir a vegetação.

\section{REFERÊNCIAS}

AGUIAR, L. M. S.; MACHADO, R. B.; MARINHO-FILHO, J. A diversidade biológica do Cerrado. In: AGUIAR, L. M. S.; CAMARGO, A. J. A. (Orgs). Cerrado ecologia e caracterização. Brasília: Embrapa Informações Tecnológicas, 2004. 249p. 
ALHO, C. J. R. Distribuição da fauna num gradiente de recurso em mosaico. In: PINTO, M.N. (Org). Cerrado: caracterização, ocupação e perspectivas. Brasília: Universidade de Brasília, 1990. 657p.

ASSUNÇÃO, S. L.; FELFILI, J. M. Fitossociologia de um fragmento de cerrado sensu stricto na APA do Paranoá, DF, Brasil. Acta Botanica Brasilica, v.18, p.903-909, 2004.

CARVALHO, A. R. Fitossociologia e modelo de distribuição de espécies em floresta ombrófila densa degradada por mineração, Joinville/ SC. Revista Saúde e

Ambiente, v.4, p.42-51, 2003.

COUTINHO, L. M. O bioma do cerrado. In: KLEIN, A. L. Eugen warming e o cerrado brasileiro. São Paulo: Universidade Estadual de São Paulo, 2000.156p.

CRONQUIST, A. An integrated system of classification of flowering plants. New York: Columbia University Press, 1981. 1262p.

DINIZ-FILHO, J. A. et al. Spatial patterns in species richness and priority areas for conservation of anurans in the Cerrado region, Central Brazil. Amphibia-

Reptilia, v.25, p.63-75, 2004.

EITEN, G. Vegetação do cerrado. In: PINTO, M. N. (Org). Cerrado: caracterização, ocupação e perspectiva. Brasília:

Universidade de Brasília, 1994. 681p.

FELFILI, J. M.; VENTUROLI, F. Tópicos em análise de vegetação. Comunicações Técnicas Florestais, v.2, n.2, p.1-25, 2000.

FELFILI, J. M. et al. Composição florística e fitossociológica de um cerrado sensu stricto em Água Boa - MT. Acta Botanica Brasilica, v.16, p.103-112, 2002.

FERREIRA, H. D. et al. Fitossociologia de um campo sujo no parque Nacional das Emas, Estado de Goiás. In: CONGRESSO NACIONAL DE BOTÂNICA, 44.,1993, São Luis. Resumos... São Luis: SBB/UFMA, 1993.
FIDELIS, A. T.; GODOY, S. A. P. Estrutura de um cerrado strico sensu na Gleba Cerrado Pé-deGigante, Santa Rita do Passa Quatro, SP. Acta Botanica Brasilica, v.17, n.4, p.531-539, 2003.

FRANCO, T. L. Levantamento florístico e síndromes de dispersão da vegetação lenhosa de do cerrado no campus da Universidade Estadual de Goiás, Anápolis. Monografia (Graduação em Ciências Biológicas) - Universidade Estadual de Goiás, Anápolis, 2004.

GENTRY, A. H. Patterns of neotropical plant species diversity. Evolutionary Biology, v.15, p.1-84, 1982.

GOODLAND, R.; FERRI, M. G. Ecologia do cerrado. São Paulo: Itatiaia, 1979. v.52. 193p.

GRAY, J. S. Species abundace patterns. In: GEE, J. H. R.; GILLER, P. S. Organization of communities: Past and present. Oxford: Blacwell Scientific, 1987. 197p.

HERINGER, E. P.; FERREIRA, M. B..

Informações preliminares a acerca da floração precoce de vinte espécies arbóreas do cerrado do Planalto Central. In: CONGRESSO NACIONAL DE BOTÂNICA, 25,. 1974, Mossoró. Anais... Mossoró:, SBB, 1974. p.2-17.

MACHADO, R. A. et al. Análise comparada da riqueza de anuros entre duas áreas com diferentes estados de conservação no município de Londrina, Paraná, Brasil (Amphibia, Anura).

Revista Brasileira de Zoologia, v.16, n.4, p.997-1004, 1999.

MAGURRAN, A. E. Ecological diversity and its measurement. Princeton: University Press, New Jersey: Chapman and Hall, 1988.179p.

MARRIS, E. The forgotten ecosystem. Nature, v.437, n.13, p.944-945, 2005.

NASCIMENTO, M. T.; SADDI, N. Structure and floristic composition in area of cerradão in Cuiabá-MT, Brazil. Revista Brasileira de Botânica, v.5, n.1, p.47-55, 1992.

NÓBREGA, R. C.; ENCINAS, J. I. Uso atual do solo do projeto ecomuseu do cerrado. Revista Árvore, v.30, n.1, p.117-122, 2006.

R. Árvore, Viçosa-MG, v.32, n.1, p.81-90, 2008 
PAGANO, S. N.; LEITÃO FILHO, H. F.;

CAVASSAN, O. Variação temporal de composição e estrutura fitossociológica de uma floresta mesófila semidecídua- Rio Claro- Estado de São Paulo. Revista Brasileira de Biologia, v.55, p.241-258, 1995.

PETRERE, M.; GIORDANO, L. C.; MARCO JR., P. Empirical diversity index applied to Forest communities in different successional stages. Brazilian Journal of Biology, v.64, p.841-851, 2004.

RATTER, J., T. C. D. DARGIE. An analysis of the floristic composition of 26 cerrado areas in Brazil. Edinburg Journal of Botany, v.49, n.2, p.235-250, 1992.

RIBEIRO, J. F. Comparação da concentração de nutrientes na vegetação e no solo de cerrado e cerradão no Distrito Federal. 1983. $87 \mathrm{f}$. Dissertação (Mestrado em Biologia) -

Universidade de Brasília, Brasília, 1983.

RIBEIRO, J. F.; SILVA, J. C. S.; BATMANIAN, G. J. Fitossociologia de tipos fisionômicos de Cerrado em Planaltina (DF). Revista Brasileira de Botânica,v.8, p.131-142, 1985.

RIBEIRO, J. F.; WALTER, B. M. T. Fitofisionomias do bioma cerrado. In: SANO, S. M.; ALMEIDA, S. P. Cerrado: ambiente e flora. Planaltina: Embrapa- CPAC, 1998. p.289-556.

ROCHA, A. E.; MUNIZ, F. H. Florística e fitossociologia da vegetação ciliar do rio Munim no município de Morros- MA. Pesquisa em Foco, v.6, p.47-65, 1998.
SAPORETTI JR, A. W.; MEIRA NETO, J. A. A.; ALMADO, R. P. Fitossociologia de cerrado sensu stricto no município de Abaeté-MG. Revista Árvore, v.27, p.413-419, 2003.

SILVA, L. O. et al. Levantamento Florístico e Fitossociológico em duas áreas de Cerrado sensu stricto no Parque Estadual da Serra de Caldas Novas, Goiás. Acta Botanica Brasilica, v.16, p.43-53, 2002.

TEIXEIRA, M. I. J. G. et al.. Florística e fitossociologia de área de cerrado sensu stricto. no município de Patrocínio Paulista, nordeste do Estado de São Paulo. Bragantia, v.63, n.1, p.1-11, 2004.

UGLANG, K. I.; GRAY, J. S. Lognormal distribution and the concept of community equilibrium. Oikos, v.39, p.171-178, 1982.

van den BERG, E.; OLIVEIRA FILHO, A. T. Composição floristíca e estrutura fitossociológica de uma floresta ripária em Itutinga MG, e comparação com outras áreas. Revista Brasileira de Botânica, v.23, p.231-253, 2000.

WATT, A. D. Measuring disturbance in tropical forests:a critique of the use of speciesabundance models and indicator measures in general. Journal of Applied Ecology, v.35, p.467-469, 1998.

WEISER, V. L.; GODOY, S. A. P. Florística em um hectare de cerrado stricto sensu na ARIECerrado Pé-de-Gigante, Santa Rita do Passa Quatro, SP. Acta Botanica Brasilica, v.15, p.201-212, 2001. 